Annuaire suisse de politique de développement

27-2 | 2008

Migration et développement: un mariage arrangé

\title{
Migration et développement : quelques portes d'entrée sur Internet
}

Martine Schaer et Chantal Hebeisen

\section{(2) OpenEdition}

Édition électronique

URL : http://journals.openedition.org/aspd/522

DOI : 10.4000/aspd.522

ISSN : 1663-9669

Éditeur

Institut de hautes études internationales et du développement

Édition imprimée

Date de publication : 1 décembre 2008

Pagination : 263-271

ISBN : 978-2-940415-07-6

ISSN : 1660-5934

Référence électronique

Martine Schaer et Chantal Hebeisen, « Migration et développement : quelques portes d'entrée sur Internet », Annuaire suisse de politique de développement [En ligne], 27-2 | 2008, mis en ligne le 22 mars 2010, consulté le 08 septembre 2020. URL : http://journals.openedition.org/aspd/522 ; DOI : https:// doi.org/10.4000/aspd.522 


\section{Migration et développement: quelques portes d'entrée sur Internet}

\section{Chantal Hebeisen et Martine Schaer*}

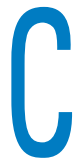

e point d'appui offre quelques portes d'entrée sur Internet aux lectrices et lecteurs souhaitant approfondir leurs connaissances sur la thématique des liens entre migration et développement. Les sites proposés ci-dessous ne constituent pas une liste exhaustive mais représentent une sélection de sites d'institutions politiques, non gouvernementales ou scientifiques, sur les plans tant suisse qu'international.

L'accent a été mis sur les sites qui concernent directement la thématique transversale du présent ouvrage, «migration et développement». Nous avons également jugé utile de mentionner quelques sites majeurs portant sur le phénomène migratoire d'une manière générale ainsi que d'autres axés sur le développement mais qui abordent de façon explicite la question migratoire ${ }^{1}$.

\section{International}

\section{Organisations internationales}

\section{$\square$ Centre international pour le développement des politiques migratoires} (ICMPD)

\section{$<$ http://www.icmpd.org > (anglais) Home > By topic}

L'ICMPD est une organisation intergouvernementale créée en 1993 par la Suisse et l'Autriche et dotée du statut d'observateur à l'ONU. L'objectif du centre est de fonctionner en tant que plate-forme d'échanges et de recherche pour le compte de gouvernements et d'organisations opérant en Europe. Il est actif dans différents domaines ayant trait aux migrations, tels que l'asile, la gestion des frontières, l'intégration, les migrations irrégulières, la question du retour et le trafic humain.

\section{$\square$ Comité international de la Croix-Rouge (CICR)}

$<$ http://www.icrc.org > (anglais, arabe, chinois, espagnol, français, portugais et russe) Home > Focus > Mouvement CR > Conseil des délégués $>2007$ > Migration

Le CICR est une organisation indépendante et neutre qui s'efforce d'apporter une protection et une assistance humanitaires aux victimes de conflits armés. Il

* Collaboratrices scientifiques au Forum suisse pour l'étude des migrations et de la population (SFM), Université de Neuchâtel.

1 Dans le cadre de ce bref tour d'horizon, il n'a malheureusement pas été possible de présenter d'une manière systématique des organismes et institutions d'autres pays, et notamment du Sud. 
est à l'origine du Mouvement international de la Croix-Rouge et du CroissantRouge ainsi que du droit international humanitaire. Le site offre notamment une rubrique sur les réfugiés et les personnes déplacées. En automne 2007, il a publié un rapport sur les migrations, document téléchargeable sur le site.

$\square$ Commission mondiale sur les migrations internationales (CMMI)

$<$ http://www.gcim.org > (anglais, espagnol et français)

La CMMI a été inaugurée à Genève en décembre 2003 par le secrétaire général des Nations unies, avec le mandat de fournir un cadre pour la formulation de réponses cohérentes aux questions liées à la migration internationale. La commission a rendu son rapport en octobre 2005. Le rapport, dont un chapitre est consacré au thème «migration et développement», est accessible en ligne depuis le site.

\section{$\square$ Haut-Commissariat des Nations unies pour les réfugiés (UNHCR)}

$<$ http://www.unhcr.org > (anglais et français) Home > Search Refworld

Le site présente l'UNHCR, et plus particulièrement ses institutions, son histoire et ses activités de recherche et de parrainage. De très nombreux documents sont accessibles en ligne. Par ailleurs, le site offre un lien vers Refworld, une base de données qui recense des informations relatives à la question des réfugiés et de l'asile dans différentes régions du monde.

$\square$ Organisation de coopération et de développement économiques (OCDE)

$<$ http://www.oecd.org > (anglais et français) Home > Par thème > Société $>$ Migrations internationales

Le site présente les activités et les thèmes dont s'occupe l'OCDE, qui regroupe 30 pays membres. En étudiant les changements et l'évolution de la société, l'organisation offre aux gouvernements un cadre leur permettant de comparer des problèmes communs, d'identifier des pratiques et de coordonner certaines politiques. Elle offre un accès aux données statistiques, économiques et sociales concernant les pays membres et une sélection de pays non membres. Des analyses détaillées sur les aspects économiques et sociaux des migrations y sont publiées.

$\square$ Organisation de coopération et de développement économiques (OCDE)Centre de développement

$<$ http://www.oecd.org/dev> (anglais et français) Home >Présentation du rapport «Migrations et pays en développement»

Le Centre de développement de l'OCDE joue un rôle d'interface entre les pays membres de l'OCDE et les pays en développement ou en transition. Il alimente le débat politique sur le développement par des analyses d'experts. En février 2008 , le centre a publié un rapport intitulé La cohérence des politiques au service du développement. Migrations et pays en développement. Une synthèse de l'ouvrage ainsi que la table des matières, la préface et l'introduction sont disponibles en ligne. Cette page référence également différentes publications de l'OCDE sur le thème de la migration. 
$\square$ Organisation internationale pour les migrations (OIM)

$<$ http://www.iom.int> (anglais, espagnol et français)

L'OIM est une organisation intergouvernementale active dans le domaine des migrations et coopérant étroitement avec des partenaires gouvernementaux comme non gouvernementaux. Elle est en charge de gérer et de promouvoir la coopération internationale sur le plan migratoire. "Migration et développement» constitue un des quatre piliers de la gestion des migrations dont s'occupe l'OIM. Le site présente les grands axes de la question migratoire et décrit les activités de l'organisation. Il donne également des informations sur l'actualité et permet d'accéder aux références des publications de l'OIM, dont certaines sont téléchargeables en ligne.

\section{$\square$ United Nations - International Migration and Development}

\section{$<$ http://www.un.org/esa/population/migration> (anglais)}

International Migration and Development, qui appartient à l'Organisation des Nations unies, a organisé à deux reprises le Forum mondial sur la migration et le développement, à Bruxelles en 2007 et à Manille en 2008. Cette page offre des liens vers des forums internationaux, des dialogues de haut niveau et des symposiums sur les migrations internationales et le développement.

\section{Recherche}

\section{$\square$ Danish Institute for International Studies (DIIS)}

\section{$<$ http://www.diis.dk> (anglais et danois) Home >Migration}

Le DIIS est un institut de recherche indépendant dans le domaine des affaires internationales. Il soutient le développement de la recherche dans les pays en développement. Le site informe sur les activités de recherche de l'institut, organisées par unités de recherche et dont l'une porte en particulier sur les migrations. Le catalogue de la bibliothèque de l'institut est accessible en ligne.

$\square$ Institut de recherche pour le développement (IRD)

$<$ http://www.ird.fr > (français) Home > L'institut >Champs de recherche

$>$ Développement et mondialisation: dynamiques socio-économiques, identitaires et spatiales

L'IRD est un établissement public français à caractère scientifique et technologique. Le site présente les programmes que l'institut conduit dans les pays du Sud dans l'objectif de contribuer à leur développement. Un des six thèmes de recherche de l'institut développe les questions migratoires (déterminants, conséquences, impact des mobilités, recompositions territoriales et sociales, réseaux et diasporas, reconstructions identitaires). Le site donne également accès à diverses bases de données en ligne de documentation scientifique, cartographique et audiovisuelle. 
$<$ http://www.imis.uni-osnabrueck.de> (allemand et anglais) Home >Arbeitsschwerpunkte

L'IMIS est un institut interdisciplinaire et interdépartemental de l'Université d'Osnabrück, en Allemagne. Le site présente les domaines de recherche prioritaires de l'institut et le programme de master «International Migration and Intercultural Relations ». Depuis le site, il est également possible d'accéder au catalogue en ligne de la bibliothèque et de commander les publications.

\section{$\square$ Migration Policy Group (MPG)}

$<$ http://www.migpolgroup.com> (anglais)

Le MPG est une organisation indépendante qui offre une plate-forme de réflexion et de coopération sur des politiques migratoires au sein de l'UE. Son objectif est de contribuer à fournir des réponses innovantes et efficaces aux défis posés par la migration et la diversité culturelle. Par ailleurs, il s'engage en faveur d'une politique de développement de la mobilité, de la migration, de la diversité, de l'égalité et de l'antidiscrimination par la facilitation des échanges entre les acteurs de tous les secteurs de la société. Des publications du MPG sont téléchargeables en ligne.

\section{$\square$ Migration Policy Institute (MPI)}

$<$ http://www.migrationpolicy.org > (anglais) Home $>$ Migration and development

Le MPI est un think tank indépendant dans le domaine de l'étude des migrations dans le monde, basé à Washington DC, aux Etats-Unis. Le site présente les activités de l'institut en fonction de cinq principaux axes de recherche, parmi lesquels figure le thème de la migration et du développement. Les publications du MPI peuvent être commandées en ligne.

\section{$\square$ Migrinter}

$<$ <ttp://www.mshs.univ-poitiers.fr/migrinter> (anglais, espagnol et français)

Migrinter est une équipe de recherche spécialisée dans l'étude des migrations internationales et des relations interethniques, hébergée par la Maison des sciences de l'homme et de la société de Poitiers, en France. Le site présente les activités de recherche développées par Migrinter, de même que ses activités de formation, de publication et de documentation. Le catalogue de son centre de documentation est accessible en ligne depuis le site.

\section{$\square$ Recherches et applications de financements alternatifs au développement (RAFAD) \\ $<$ http://www.rafad.org > (anglais, espagnol et français) Home > Transferts de fonds (Remittances)}

RAFAD est une fondation privée internationale dont le rôle est de conseiller, d'encadrer et d'appuyer les organisations locales des pays du Sud soutenant les micro et petites entreprises afin qu'elles puissent consolider leurs processus de pérennisation. Les migrants participent ainsi au développement de leur pays 
d'origine en envoyant de l'argent à leur famille. La fondation a développé une expertise dans le domaine des remittances pour aider les institutions de microfinance à étudier et développer leur marché. Une bibliothèque virtuelle est actuellement en construction.

\section{$\square$ Sussex Centre for Migration Research}

$<$ http://www.sussex.ac.uk/migration> (anglais) Home $>$ Research

$>$ Development Research Centre on Migration, Globalisation and Poverty

Le Sussex Centre for Migration Research est un département de l'Université du Sussex. Il mène des activités de recherche et de formation dans le domaine du développement et de la migration par le biais du Development Research Centre on Migration, Globalisation and Poverty. De nombreux documents téléchargeables sont disponibles sur le site.

\section{Suisse}

\section{Offices fédéraux et autres institutions}

$\square$ Commission fédérale pour les questions de migration (CFM) $<$ <ttp://www.eka-cfe.ch/(allemand, anglais, français et italien) Home > Thèmes

La Commission fédérale pour les questions de migration est une commission d'experts du Conseil fédéral; elle remplace la Commission fédérale des étrangers et la Commission fédérale des réfugiés. La CFM traite des questions sociales, économiques, culturelles, politiques, juridiques et démographiques. Elle prend notamment position sur les questions de migration et d'intégration. De nombreux documents sont téléchargeables sur l'ancien site de la Commission fédérale des étrangers.

$\square$ Direction du développement et de la coopération (DDC)

$<$ http://www.ddc.admin.ch> (allemand, anglais, espagnol, français et italien) Home $>$ Thèmes $>$ Migrations

La DDC est l'organe fédéral en charge de la coopération internationale au développement. Parmi les thèmes mentionnés sur son site figure la migration, dont les risques et potentiels sont analysés. Le site traite également des thématiques de l'aide au retour et du trafic d'humains. Une importante documentation est disponible en ligne.

\section{$\square$ Division politique IV, Sécurité humaine - Département fédéral des affaires étrangères (DFAE) \\ $<$ http://www.eda.admin.ch> (allemand, anglais, français et italien) Home $>$ Le DFAE > Organigramme > Directions et divisions $>$ Division politique IV}

La Division politique IV du Département fédéral des affaires étrangères (DFAE) est en charge de la mise en œuvre des objectifs de promotion civile de la paix et du renforcement des droits de l'homme. Elle a organisé en automne 2007 une conférence intitulée «La migration - Opportunités et défis». La documentation de la conférence est disponible en ligne. 
$\square$ Office fédéral des migrations (ODM)

$<$ http://www.bfm.admin.ch> (allemand, anglais, français et italien) Home $>$ Thèmes

L'Office fédéral des migrations traite de toutes les questions relevant du droit des étrangers et du droit d'asile en Suisse. Le site donne accès à divers thèmes concernant les migrations, dont l'aide au retour et la réintégration, que l'ODM développe pour certains pays en collaboration avec la Direction du développement et de la coopération (DDC) et l'Organisation internationale pour les migrations (OIM). De nombreux documents sont téléchargeables sur le site Internet.

$\square$ Organisation internationale pour les migrations (OIM) - Antenne suisse $<$ http://www.ch.iom.int> (allemand et français) Home >Activités

L'OIM est une organisation intergouvernementale active mondialement dans le domaine de la migration. L' ' OIM Berne» est le bureau de contact et de coordination pour les questions nationales et internationales relatives à la migration en Suisse. Le site consacre une partie de ses activités à l'aide au retour, à l'immigration vers un pays tiers ainsi qu'au regroupement familial. Les publications peuvent être commandées en ligne.

$\square$ Secrétariat d'Etat à l'économie (SECO) - Centre de prestations Coopération et Développement économiques

$<$ http://www.seco-cooperation.admin.ch> (allemand, anglais, français et italien)

Le centre de prestations Coopération et Développement économiques du SECO est chargé, conjointement avec la Direction du développement et de la coopération (DDC), de coordonner la coopération de la Suisse avec les pays en développement, les pays d'Europe de l'Est et d'Asie centrale et les dix nouveaux Etats membres de l'Union européenne (UE). Ses objectifs sont l'intégration des pays dans l'économie mondiale et la promotion de leur croissance économique afin de réduire la pauvreté. Depuis cette page, il est possible de télécharger des rapports et de commander des brochures gratuites.

\section{Organisations non gouvernementales \\ actives dans le domaine "migration et développement»}

\section{$\square$ Caritas Suisse}

$<$ http://web.caritas.ch> (allemand, anglais et français) Home $>$ Suisse $>$ Migration/Asile

Caritas Suisse est une association d'entraide faisant partie du réseau des Caritas, présent dans plus de 200 pays. Sur le site sont présentés les parrainages et les divers domaines dans lesquels l'organisation est active, notamment celui de la migration et de l'asile. Les publications de Caritas sont téléchargeables en ligne. 
$<$ http://www.redcross.ch> (allemand, anglais, français et italien) Home $>$ Intégration $>$ Migration

La Croix-Rouge suisse est le membre local du Mouvement international de la Croix-Rouge et du Croissant-Rouge. Elle a pour but de protéger la vie, la santé et la dignité des personnes en détresse. Sur le site de l'organisation suisse, on trouve une section relative à la migration en Suisse, et en particulier aux questions d'aide au retour et de formation. Du matériel d'information sur cette thématique est disponible en ligne.

$\square$ Entraide protestante suisse (EPER)

$<$ http://www.eper.ch> (allemand et français)

L'EPER est l'œuvre d'entraide des Eglises protestantes suisses. Elle apporte une aide d'urgence et de survie afin de pouvoir améliorer les conditions de vie de manière durable. Dans le cadre de son engagement en faveur des réfugiés et des personnes déplacées, l'EPER conduit notamment des projets d'intégration en Suisse et dans les pays en développement. Le site renseigne sur les activités et les priorités de l'organisation.

\section{$\square$ Fédération genevoise de coopération (FGC)}

$<$ http://www.fgc.ch> (allemand, anglais, espagnol et français) Home $>$ Actualités $>$ Forums

La FGC jour le rôle d'interface entre les associations membres et les collectivités publiques genevoises. Dans l'idée d'un espace de dialogue et d'échanges, elle travaille avec ses partenaires pour combattre les inégalités dans le monde et augmenter la liberté de choix des sociétés et des peuples dans leur construction d'un mieux-être économique, social et culturel. Un forum sur le thème «projets de développement FGC et migrations » a eu lieu en juin 2008.

\section{$\square$ Fédération vaudoise de coopération (FEDEVACO)}

$<$ http://www.fedevaco.ch> (français)

La FEDEVACO, en tant qu'organisation faîtière, joue le rôle de plate-forme d'échanges entre ses associations membres et les collectivités publiques vaudoises. Elle cherche des financements institutionnels pour les projets de ses membres et promeut un dialogue et une «réciprocité» Nord-Sud sur des thématiques particulières. Les trois thèmes prioritaires, définis avec les autorités cantonales, sont les suivants: «santé publique», «développement durable (changements climatiques)» et «migration et développement».

\section{$\square$ Forum pour l'intégration des migrantes et des migrants (FIMM)}

$<$ http://www.fimm-integration.ch> (allemand et français)

Le FIMM Suisse est l'organisation faîtière des associations de migrants en Suisse. Les communautés de plus de 50 nationalités y sont représentées. Le site trace un bref portrait de l'organisation et présente ses objectifs et axes stratégiques. Le texte de la Charte de l'intégration du FIMM Suisse est téléchargeable sur le site et disponible en allemand, français et italien. 
$\square$ Humanrights.ch - Association suisse pour les droits humains (MERS)

$<$ http://www.humanrights.ch> (allemand, anglais et français) Home

$>$ Nos services $>$ Thèmes de A à $Z>$ Droit et politique d'asile et des réfugiés et $>$ Droit et politique des étrangers

L'Association suisse pour les droits humains promeut, réalise et coordonne des projets en relation avec les droits humains. Une attention particulière est portée à la situation des migrants, des demandeurs d'asile et des sans-papiers. De nombreux liens vers d'autres acteurs de la défense des droits humains ainsi que de la documentation sont disponibles sur le site (>Nos services $>$ Liens choisis dans le domaine des droits humains > Scène des droits humains en Suisse >Migration, asile, étranger-e-s).

\section{$\square$ Maison Kultura}

$<$ http://www.kultura.ch> (français)

La Maison Kultura est une organisation faîtière regroupant une quarantaine d'organisations (asiatiques, africaines, européennes et latino-américaines) actives sur le terrain de l'immigration, l'interculturalité et la coopération internationale de la région genevoise. Elle fonctionne en tant que carrefour de rencontres, d'entraide et de dialogue.

\section{$\square$ Organisation suisse d'aide aux réfugiés (OSAR)}

$<$ http://www.osar.ch> (allemand, anglais et français) Home >Adresses/Liens

L'OSAR est l'organisation faîtière des œuvres d'entraide suisses actives dans l'aide aux réfugiés. Le site informe de manière détaillée sur le droit d'asile, la politique d'asile suisse et la situation dans les pays d'origine des réfugiés. Par ailleurs, il propose des liens et des adresses divers (bureaux de consultation juridique, membres et partenaires de l'OSAR). Une partie des publications de l'OSAR peut être téléchargée directement depuis le site.

\section{$\square$ Solidarité sans frontières (SOSF)}

\section{$<$ http://www.sosf.ch> (allemand et français) Home > Migration}

SOSF est une organisation indépendante d'information dans les domaines de la politique d'asile et migratoire, du racisme et des droits humains. Elle collabore directement avec les personnes touchées. Elle lutte pour la bonne entente entre tous, indépendamment de l'origine de chacun et dans l'égalité des droits, et contre la xénophobie et le racisme. De nombreux articles sont téléchargeables sur le site.

\section{$\square$ Swiss Academy for Development (SAD) $<$ http://www.sad.ch> (allemand, anglais et français) Home >Domaine $>$ Dialogue interculturel}

La SAD est une fondation d'utilité publique active dans la conception et la réalisation de solutions et de stratégies durables dans le domaine de la coopération au développement et de l'intégration. Soutenant l'intégration et la participation des groupes culturels, la SAD a réalisé des études scientifiques et des projets axés sur la pratique de la cohabitation au sein de sociétés pluriculturelles. Les publications sont téléchargeables depuis le site. 
$\square$ Terre des hommes (TDH)

$<$ http://www.tdh.ch> (allemand, anglais, français, italien) Home > Thèmes $>$ Suisse

Terre des hommes est une fondation qui poursuit des objectifs d'information et d'amélioration des conditions de vie, notamment des enfants et des femmes, dans les pays du Sud, cela grâce à des programmes humanitaires. Elle lutte notamment contre la traite d'êtres humains et le trafic des migrants. Les prises de position peuvent être consultées sur le site Internet.

\section{Formation et recherche}

\section{$\square$ Fondation Education et Développement (FED)}

$<$ http://www.globaleducation.ch> (allemand, anglais, français et italien)

La Fondation Education et développement est le centre de compétence national sur l'éducation dans une perspective globale. Elle promeut un enseignement qui encourage les enfants et les jeunes à agir de manière responsable dans la société mondiale. Elle s'adresse en premier lieu aux enseignants, mais également aux chercheurs, aux experts et aux responsables dans le domaine de l'éducation. Le site informe sur le matériel pédagogique et les différentes offres de formation qu'elle propose et donne un aperçu des campagnes et projets, en lien avec les migrations notamment, dans lesquels elle s'engage.

\section{$\square$ Forum suisse pour l'étude des migrations et de la population (SFM)}

\section{$<$ http://www.migration-population.ch> ( allemand, anglais et français)}

Le SFM est un institut de l'Université de Neuchâtel qui effectue des travaux de recherche dans le domaine des migrations et des questions démographiques. Le site donne un descriptif des publications de l'institut, dont la plupart sont téléchargeables en ligne. Un lien permet d'accéder au catalogue en ligne de son centre de documentation.

$\square$ Institut de hautes études internationales et du développement (IHEID) $<$ http://www.graduateinstitute.ch> (anglais et français) Home $>$ Recherche $>$ Programme for the Study of Global Migration

L'institut est issu du regroupement de l'Institut universitaire de hautes études internationales (HEI) et de l'Institut universitaire d'études du développement (IUED). Le site présente les activités d'enseignement et de recherche de l'institut. En janvier 2008 a été créé le «Programme for the Study of Global Migration», qui comprend des études interdisciplinaires sur les migrations économiques. Le programme se concentre sur les thèmes des conflits armées, la violence généralisée et la persécution ainsi que sur les désastres écologiques. Des publications sont téléchargeables sur le site. 